\title{
The 2016 WHO classification and diagnostic criteria for myeloproliferative neoplasms: document summary and in- depth discussion
}

\author{
Tiziano Barbui ${ }^{1}$, Jürgen Thiele ${ }^{2}$, Heinz Gisslinger ${ }^{3}$, Hans Michael Kvasnicka ${ }^{4}$, Alessandro M. Vannucchi ${ }^{5}$, \\ Paola Guglielmelli ${ }^{6}{ }^{6}$, Attilio Orazi ${ }^{7}$ and Ayalew Tefferi $^{8}$
}

\begin{abstract}
The new edition of the 2016 World Health Organization (WHO) classification system for tumors of the hematopoietic and lymphoid tissues was published in September 2017. Under the category of myeloproliferative neoplasms (MPNs), the revised document includes seven subcategories: chronic myeloid leukemia, chronic neutrophilic leukemia, polycythemia vera (PV), primary myelofibrosis (PMF), essential thrombocythemia (ET), chronic eosinophilic leukemianot otherwise specified and MPN, unclassifiable (MPN-U); of note, mastocytosis is no longer classified under the MPN category. In the current review, we focus on the diagnostic criteria for JAK2/CALR/MPL mutation-related MPNs: PV, ET, and PMF. In this regard, the 2016 changes were aimed at facilitating the distinction between masked PV and JAK2mutated ET and between prefibrotic/early and overtly fibrotic PMF. In the current communication, we (i) provide practically useful resource tables and graphs on the new diagnostic criteria including outcome, (ii) elaborate on the rationale for the 2016 changes, (iii) discuss the complementary role of mutation screening, (iv) address ongoing controversies and propose solutions, ( $v$ ) attend to the challenges of applying WHO criteria in routine clinical practice, and (vi) outline future directions from the perspectives of the clinical pathologist.
\end{abstract}

\section{Introduction}

The 2016 revised "Blue Book", the official document of the World Health Organization (WHO) classification system for tumors of the hematopoietic and lymphoid tissues, has now been published ${ }^{1}$. The current communication focuses on myeloproliferative neoplasms (MPNs) and provides a more comprehensive syllabus that is organized into eight sections: section one starts with a list and brief overview of the seven clinic-pathologic entities that currently comprise the WHO MPN category; section two provides practically useful resource tables and graphs on the 2016 WHO diagnostic criteria and outcome for the

\footnotetext{
Correspondence: Tiziano Barbui (tbarbui@asst-pg23.it)

${ }^{1}$ FROM Research Foundation, Papa Giovanni XXIII Hospital, Bergamo, Italy

${ }^{2}$ Institute of Pathology, University of Cologne, Cologne, Germany
}

Full list of author information is available at the end of the article
JAK2/CALR/MPL mutation-related MPNs, including polycythemia vera (PV), essential thrombocythemia (ET), and primary myelofibrosis (PMF), including particularly prefibrotic/early PMF (pre-PMF); section three addresses the rationale behind the 2016 changes in the diagnostic criteria for PV, ET, PMF; section four attends to the complementary role of mutation screening and its limitations for diagnostic purposes; section five highlights current controversies regarding the new diagnostic criteria, especially in regards to diagnosis of PV and prePMF; section six offers proposed solutions for currently ongoing controversies; section seven considers the challenges in applying the WHO criteria in routine clinical practice, and discusses future directions from the perspective of the physician scientist; section eight outlines 
solutions and future directions from the perspective of the clinical pathologist.

\section{The 2016 WHO sub-categorization of MPNs and brief overview of the diagnostic criteria for CML, CNL, CEL-NOS, and MPN-U}

Morphology remains the central distinguishing feature in the 2016 WHO system for classification of tumors of the hematopoietic and lymphoid tissues, although mutation screening is increasingly being utilized for confirmation of morphologic diagnosis and, at times, for directing the diagnostic process ${ }^{1,2}$.

Myeloid neoplasms continue to be organized into acute myeloid leukemia and chronic myeloid neoplasms, based primarily on the percentage of peripheral blood or bone marrow (BM) blasts. Chronic myeloid neoplasms are in turn classified into four operational categories: myelodysplastic syndromes (MDS), MPNs, MDS/MPN overlap and myeloid/lymphoid neoplasms with eosinophilia and recurrent rearrangements of PDGFRA, PDGFRB, and FGFR1 or PMC1-JAK2; the latter mutations correspond to $5 \mathrm{q} 33,4 \mathrm{q} 12,8 \mathrm{p} 11.2$ or $\mathrm{t}(8 ; 9)(\mathrm{p} 22 ; \mathrm{p} 24.1)$ cytogenetic abnormalities, respectively. MPNs are generally distinguished from both MDS and MDS/MPN, by the absence of morphologic dysplasia, which includes dyserythropoiesis and dysgranulopoiesis and monocytosis.

The 2016 WHO category of MPNs includes the three major subcategories of JAK2/CALR/MPL mutationrelated MPNs (i.e., PV, ET, and PMF), as well as four other clinicopathologic entities: chronic myeloid leukemia (CML), chronic neutrophilic leukemia (CNL), chronic eosinophilic leukemia, not otherwise specified (CELNOS) and MPN, unclassifiable (MPN-U). The JAK2/ CALR/MPL mutation-related MPNs constitute the main focus of discussion in the current review and are further elaborated in sections 2 through $8^{1,2}$.

The diagnostic hallmark of CML is the invariable presence of the $B C R-A B L 1$ mutation. However, minor $B C R$ $A B L 1$-harboring sub-clones are sometimes detected in other myeloid neoplasms, including the JAK2/CALR/ $M P L$-mutated MPNs, and do not necessarily alter the morphologically prominent diagnosis ${ }^{3}$. Similarly, JAK2mutated clones are sometimes detected in patients with CML, especially after successful treatment with imatinib ${ }^{4}$.

$\mathrm{CNL}$ constitutes clonal proliferation of mature neutrophils and is usually associated with activating mutations (mostly T618I) of the gene (CSF3R) encoding for the receptor for granulocyte colony-stimulating factor, also known as colony-stimulating factor $3^{5}$. CSF3R mutations appear to be specific to WHO-defined $\mathrm{CNL}^{6}$. Diagnosis of $\mathrm{CNL}$ requires exclusion other causes of neutrophilia, including infections and inflammatory processes, metastatic cancer, and plasma cell neoplasms with secondary neutrophilia ${ }^{7}$. Mature-appearing neutrophilia also occurs in other myeloid malignancies, including atypical CML, $B C R-A B L 1$-negative (aCML) and chronic myelomonocytic leukemia. Accordingly, the 2016 WHO diagnostic criteria for $\mathrm{CNL}$ are designed to exclude the possibilities of both secondary and clonal neutrophilia associated with myeloid malignancies other than CNL: leukocytosis $\left(\geq 25 \times 10^{9} / \mathrm{L}\right), \geq 80 \%$ segmented/band neutrophils, $<10 \%$ immature myeloid cells, $<1 \%$ circulating blasts and absence of dysgranulopoiesis or monocytosis (monocyte count $<1 \times 10^{9} / \mathrm{L}$ ). In clinical practice, the presence of a membrane proximal CSF3R mutation in a patient with neutrophilic granulocytosis should be sufficient for the diagnosis of CNL, regardless of the degree of leukocytosis.

CEL-NOS constitutes clonal eosinophilia and is considered in the presence of $\geq 1.5 \times 10^{9} / \mathrm{L}$ absolute eosinophil count in the peripheral blood that is accompanied by either the presence of myeloblast excess (either $>2 \%$ in the peripheral blood or $5-19 \%$ in the bone marrow) or presence of a clonal cytogenetic abnormality ${ }^{8}$. Cytogenetic abnormalities in CEL-NOS include trisomy 8 (the most frequent), $t(10 ; 11)(\mathrm{p} 14 ; \mathrm{q} 21)$, and $\mathrm{t}(7 ; 12)(\mathrm{q} 11 ; \mathrm{p} 11)$. Targeted next-generation sequencing studies have recently suggested the possibility of re-classifying some cases of "hypereosinophilic syndrome" as CEL-NOS $9,10$. Unlike the case with PDGFRA/B-rearranged myeloid/ lymphoid neoplasms with eosinophilia, imatinib therapy is ineffective in CEL-NOS.

The WHO MPN sub-category of MPN-U includes MPN-like neoplasms that cannot be clearly classified as one of the other six subcategories of MPNs. Patients with MPN-U might present with otherwise unexplained thrombosis, especially splanchnic vein thrombosis ${ }^{11}$, which is associated with normal blood count.

\section{Practically useful resource tables and graphs on the 2016 WHO diagnostic criteria for PV, ET, and PMF including particularly pre-PMF}

The combination of clinical, morphological, and molecular genetic features is thought by the WHO as the most suitable attempt to define disease entities such as MPNs (Tables 1 and 2) 1,2, 12 . Following the updated $2008 \mathrm{WHO}$ classification $^{12}$, a number of clinical-pathological studies conducted by different groups have validated these diagnostic guidelines including the importance of morphological features ${ }^{13-22}$. However, a balanced and evidencebased discussion concerning these diagnostic criteria persists $^{23}$. In particular, it has been postulated that ET, $\mathrm{PV}$, and PMF cannot be strictly discriminated by BM morphology as postulated by the WHO, owing to their mimicry to transform to each other ${ }^{24,25}$. It was argued that JAK2-mutated ET resembles PV for similarities of hematological presentation and incidence of clinical manifestations. It is important that this notion should be revisited as the results refer to patients diagnosed with not 
Table 12016 World Health Organization diagnostic criteria for polycythemia vera and essential thrombocythemia

\begin{tabular}{|c|c|c|}
\hline & Polycythemia vera $(P V)^{a}$ & Essential thrombocythemia $(E T)^{\mathbf{b}}$ \\
\hline \multicolumn{3}{|c|}{ Major criteria } \\
\hline & $\begin{array}{l}\text { Hemoglobin }>16.5 \mathrm{~g} / \mathrm{dL} \text { (men)Hemoglobin }>16.0 \mathrm{~g} / \mathrm{dL} \text { (women)or } \\
\text { Hematocrit }>49 \% \text { (men) Hematocrit }>48 \% \text { (women) orincreased red } \\
\text { cell mass }(\mathrm{RCM})^{c}\end{array}$ & Platelet count $\geq 450 \times 10^{9} / \mathrm{L}$ \\
\hline & $\begin{array}{l}\text { BM biopsy showing hypercellularity for age with trilineage growth } \\
\text { (panmyelosis) including prominent erythroid, granulocytic and } \\
\text { megakaryocytic proliferation with pleomorphic, mature } \\
\text { megakaryocytes (differences in size) }\end{array}$ & $\begin{array}{l}\text { BM biopsy showing proliferation mainly of the megakaryocyte lineage } \\
\text { with increased numbers of enlarged, mature megakaryocytes with } \\
\text { hyperlobulated nuclei. No significant left-shift of neutrophil granulopoiesis } \\
\text { or erythropoiesis and very rarely minor (grade 1) increase in reticulin }_{\text {fibers }^{\text {d }}}\end{array}$ \\
\hline & Presence of JAK2 or JAK2 exon 12 mutation & $\begin{array}{l}\text { Not meeting WHO criteria for } B C R-A B L 1+C M L, P V, P M F, M D S \text {, or other } \\
\text { myeloid neoplasms }\end{array}$ \\
\hline 4 & & Presence of JAK2, CALR or MPL mutation \\
\hline \multicolumn{3}{|c|}{ Minor criteria } \\
\hline 1 & Subnormal serum erythropoietin level & $\begin{array}{l}\text { Presence of a clonal marker (e.g., abnormal karyotype) or absence of } \\
\text { evidence for reactive thrombocytosis }\end{array}$ \\
\hline \multicolumn{3}{|c|}{$\begin{array}{l}\text { Table adapted from Barbui T et al. Blood Cancer J 2015; } 5: e 337^{103} \text { and Arber et al. Blood 2016;127:2391-2405 } \\
\text { BM, bone marrow; CML, chronic myeloid leukemia; MDS, myelodysplastic syndrome } \\
\text { a PV diagnosis requires meeting either all three major criteria or the first two major criteria and one minor criterion } \\
\text { b ET diagnosis requires meeting all four major criteria or first three major criteria and one minor criterion } \\
\text { cMore than } 25 \% \text { above mean normal predicted value } \\
\text { d Grading of BM fibers } 87 \\
\text { Criterion number } 2 \text { (BM biopsy) may not be required in cases with sustained absolute erythrocytosis: hemoglobin levels. } 18.5 \mathrm{~g} / \mathrm{dL} \text { in men (hematocrit, } 55.5 \% \text { ) or } 16.5 \\
\mathrm{~g} / \mathrm{dL} \text { in women (hematocrit, } 49.5 \% \text { ) if major criterion } 3 \text { and the minor criterion are present. However, initial myelofibrosis (present in up to } 20 \% \text { of patients) can only } \\
\text { be detected by performing a BM biopsy; this finding may predict a more rapid progression to overt myelofibrosis (post-PV MF) }\end{array}$} \\
\hline
\end{tabular}

strictly based WHO criteria $^{2,12}$. As an example, in a cohort of 466 JAK2-mutated ET patients a cumulative risk of evolution to PV from ET was found in $29 \%$ at 15 years $^{25}$. However, when strictly adhering to the WHO criteria, the rate of transformation of ET into PV after two decades of follow-up, was rarely documented and accounted for a rate of $1 \%$ and only up to $5 \%$ of wild type and $J A K 2$-mutated ET, respectively ${ }^{26-29}$. The diagnostic differentiation between ET and pre-PMF is not only supported by characteristic morphological BM features of the two diseases but it is also highlighted by the different clinical behavior as reported in Fig. 1a-d. ET is the more benign entity in terms of survival, progression to myelofibrosis (MF) and transformation to blastic phase. Instead the cumulative incidence of major thrombosis in ET is comparable to pre-PMF and lower than PV. On the other hand, pre-PMF has a clear distinct clinical pattern of evolution from ET in terms of evolution into overt PMF, blast crisis, and mortality (Fig. 1a, c, d) and, as previously reported, increased bleeding tendency ${ }^{22}$. In PV, that in the current classification $^{1,2}$ also includes cases with a prodromal/masked phase $(\mathrm{mPV})^{30}$ there is a trend, in comparison with the other entities, to more frequent thrombotic events and of higher incidence of progression to MF. In overt PMF rates for mortality and transformation to blast crisis are the highest of all MPN subtypes under study, whereas the cumulative incidence of thrombotic complications is lower (Fig. 1a, b, d).

\section{Rationale behind the 2016 changes in the diagnostic criteria for PV, ET, PMF, and pre-PMF}

In comparison with the 2008 WHO guidelines ${ }^{12}$, several important improvements mostly derived from clinicpathological and molecular genetic studies have been highlighted:

(i) Discovery of novel molecular findings that provide deeper insights for the understanding of the pathobiology of MPNs that are in keeping with clonality $^{31}$ and exert an impact on diagnosis ${ }^{26,32}$ and outcome ${ }^{14,15,26}$.

(ii) Lowering of the diagnostic hemoglobin ( $\mathrm{Hb}) /$ hematocrit (Hct) threshold values with introduction of $\mathrm{mPV}$ that has changed markedly the diagnostic landscape of this MPN subtype and consequently options for treatment and outcome $\mathrm{e}^{30}$, ${ }^{33-35}$ by revealing that PV has been underdiagnosed in the past ${ }^{34,36}$. In this context, BM histology was promoted from a minor to a major diagnostic criterion by recognizing its reproducible characteristic morphological features ${ }^{37-40}$. 
Table 22016 World Health Organization diagnostic criteria for primary myelofibrosis

Primary myelofibrosis (PMF)

Prefibrotic/early PMF (pre-PMF)

Overt PMF

Major criteria

1 Megakaryocytic proliferation and atypia ${ }^{\mathrm{b}}$, without reticulin fibrosis > grade $1^{c}$, accompanied by increased age-adjusted BM cellularity,

Megakaryocyte proliferation and atypia ${ }^{\mathrm{b}}$ accompanied by either reticulin granulocytic proliferation and often decreased erythropoiesis

2 Not meeting WHO criteria for BCR-ABL1 + CML, PV, ET, MDS, or other myeloid neoplasm

3 Presence of JAK2, CALR, or MPL mutation or in the absence of these mutations, presence of another clonal marker ${ }^{\mathrm{d}}$ or absence of minor reactive $B M$ reticulin fibrosis ${ }^{e}$

Minor criteria

1 Presence of one or more of the following, confirmed in two consecutive determinations:

- Anemia not attributed to a comorbid condition and/or collagen fibrosis (grade 2 or 3 )

Not meeting WHO criteria for BCR-ABL1 + CML, PV, ET, MDS or other myeloid neoplasm

Presence of JAK2, CALR, or MPL mutation or in the absence, the presence of another clonal marker ${ }^{d}$ or absence of evidence for reactive BM fibrosis ${ }^{\mathrm{f}}$

- Leukocytosis $\geq 11 \times 10^{9} / \mathrm{L}$

- Palpable splenomegaly

Presence of one or more of the following confirmed in two consecutive determinations:

- Anemia not attributed to a comorbid condition

- Leukocytosis $\geq 11 \times 10^{9} / \mathrm{L}$

- Palpable splenomegaly

- LDH level above the upper limit of the institutional reference range

- LDH level above the upper limit of the institutional reference range

- Leukoerythroblastosis

Table adapted from Barbui T et al. Blood Cancer J. 2015; 5:e337 ${ }^{103}$. and Arber et al. Blood 2016;127:2391-2405²

$B M$, bone marrow; $C M L$, chronic myeloid leukemia; $M D S$, myelodysplastic syndrome; $L D H$, serum lactate dehydrogenase

${ }^{\mathrm{a}}$ Diagnosis of prefibrotic/early PMF requires all three major criteria and at least one minor criterion. Diagnosis of overt PMF requires meeting all three major criteria and at least one minor criterion

${ }^{b}$ Small-to-large megakaryocytes with aberrant nuclear/cytoplasmic ratio and hyperchromatic and irregularly folded nuclei and dense clustering

In cases with grade 1 reticulin fibrosis ${ }^{87}$, the megakaryocyte changes must be accompanied by increased BM cellularity, granulocytic proliferation, and often decreased erythropoiesis (that is, pre-PMF)

${ }^{d}$ In the absence of any of the three major clonal mutations, the search for the most frequent accompanying mutations (ASXL1, EZH2, TET2, IDH1/IDH2, SRSF2, SF3B1) are of help in determining the clonal nature of the disease

${ }^{\mathrm{e}}$ Minor (grade 1) reticulin fibrosis secondary to infection, autoimmune disorder or other chronic inflammatory conditions, hairy cell leukemia or other lymphoid neoplasm, metastatic malignancy, or toxic (chronic) myelopathies

$\mathrm{BM}$ fibrosis secondary to infection, autoimmune disorder, or other chronic inflammatory conditions, hairy cell leukemia, or other lymphoid neoplasm, metastatic malignancy or toxic (chronic) myelopathies

(iii) Emphasizing the need to discriminate "true" ET from pre-PMF by an accurate evaluation of BM biopsy features ${ }^{41}$, including the lack of reticulin fibrosis at onset in $<5 \%$ of cases, which has been formerly neglected ${ }^{42}$. It can only be underscored that this distinction is of significant prognostic and therapeutic relevance ${ }^{13,15-18}$.

(iv) Advancements regarding the characterization and standardization of morphological BM features yielded an improvement in the differentiation of MPN subtypes, particularly between ET, pre-PMF, and $\mathrm{PV}^{17,20,43,44}$. The latter presents one of the critical key issues for hemato-pathologists to improve their agreement rates (up to 80\% depending on study design) ${ }^{17-19,45,46}$ and decrease the number of unclassifiable cases (currently down to maximal $5 \%)^{47}$.

Following the 2016 revision $^{1,2}$ of the 2008 diagnostic guidelines proposed by the $\mathrm{WHO}^{12}$, critical questions were still raised and reflected by comments in recently published reviews on $\mathrm{MPNs}^{48}$, ${ }^{49}$. In one of these reviews ${ }^{48}$ these refer to the presentation of borderline expressed so-called minor clinical criteria in pre-PMF ${ }^{15}$ or the $\mathrm{Hb}$ threshold values necessary to diagnose $\mathrm{PV}^{34}$. More general arguments are related to the failing diagnostic specificity of BM morphology for differentiation of MPNs, except that myelodysplasia can be ruled out on the basis of histologic features ${ }^{49}$. Erroneously, it is assumed that the transformation of MPN demonstrates that diagnosis is a moving target ${ }^{49}$. According to the WHO classification ${ }^{1,2}$, $12 \mathrm{mPV}$ may initially mimic ET and therefore usually transforms later to overt $\mathrm{PV}^{27,50,51}$ or pre-PMF may present with an ET-like phenotype and may progress to overt $\mathrm{PMF}^{13,15,18}$. In aggregate, these so-called instabilities of subtyping MPNs are significantly dependent on the accuracy of initial diagnosis ${ }^{27}$. 


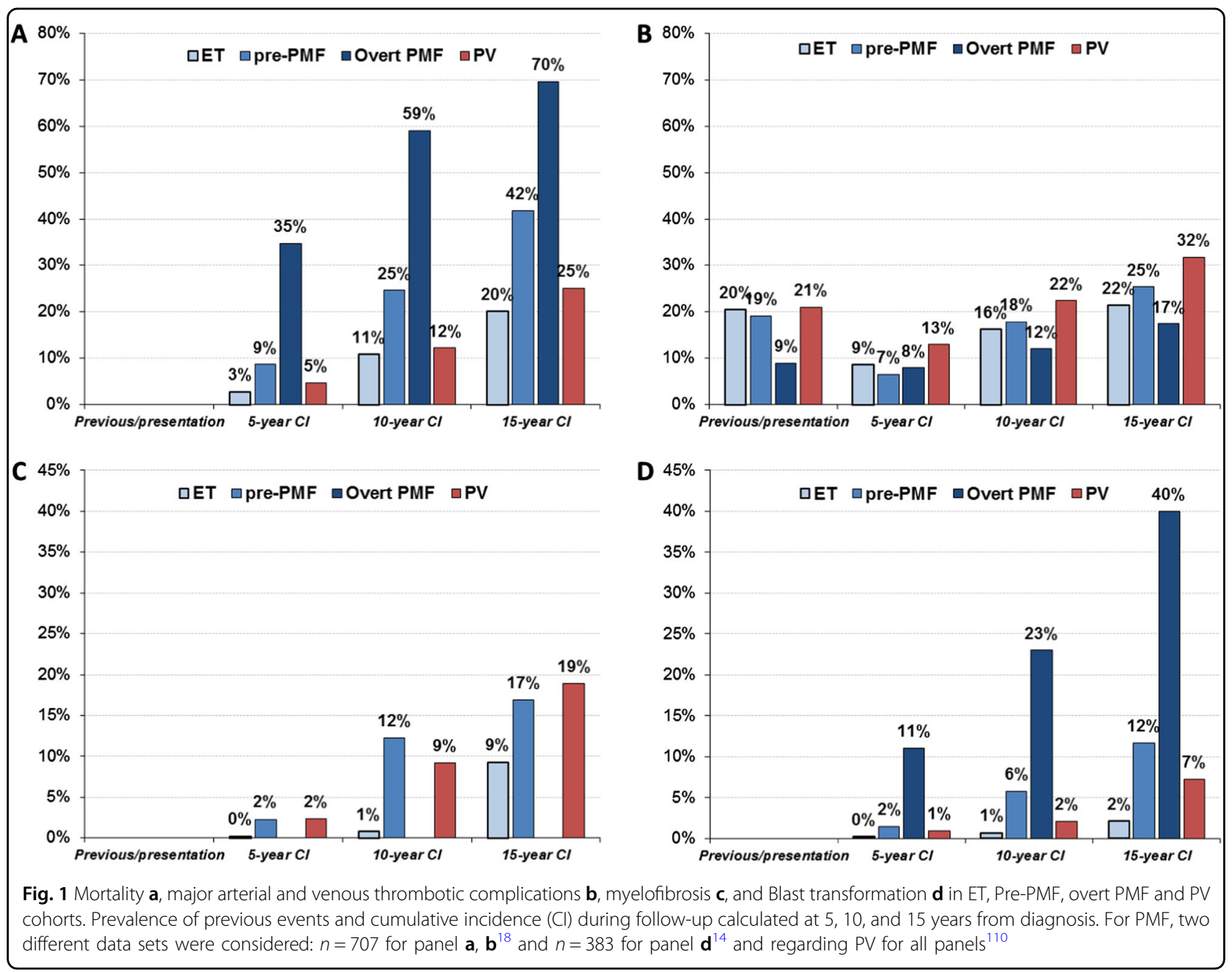

The complementary role of mutation screening and its limitations for diagnostic purposes

The 2008 WHO classification of MPN was largely inspired by the discovery of mutations in JAK2 (chr. 9p24), namely V617 $\mathrm{F}^{52-55}$ in exon 14 and indels in exon $12^{56}$, and MPL (chr. 1p34), mainly at codon W515 $5^{57}$, that were incorporated as major diagnostic criteria ${ }^{12}$. The $J A K 2 \mathrm{~V} 617 \mathrm{~F}$ is the most prevalent mutation in MPN, accounting for $\sim 95 \%$ of PV and $60 \%$ of ET and PMF. Variable deletions and insertions clustering at codon $537-543$ in exon 12 of $J A K 2$ are detected in $\sim 3-5 \%$ of patients with $J A K 2$ V617F unmutated PV by using sensitive approaches, as mutation allelic burden in whole blood and purified granulocytes is $l^{5}{ }^{58}$. Mutations in $M P L$ cluster in exon 10 at codon 515, the most prevalent being a W to K, L, A, R transversion, and rarely at codon 505 (S $>N$ ), originally reported in familial cases of thrombocytosis $^{59}$. They are found in ET and PMF with approximate incidence of 4 and $8 \%{ }^{60}$. Finally, in 2013, mutations in CALR (chr. 19p13.2), the gene encoding the endoplasmic reticulum-associated chaperone calreticulin, were detected in patients with $J A K 2 / M P L$ unmutated ET and $\mathrm{PMF}^{61,62}$. These are highly heterogeneous indels, all clustering in exon 9 that encodes for the C-terminus portion of the protein. There are two prevalent ( $>80 \%$ of all CALR variants) mutation types, type 1 (a 52-bp deletion; p.L367fs*46) and type 2 (a 5-bp insertion; p. K385fs*47), whereas the remaining are defined as type 1like and type 2-like based on predicted helix propensity similarities with the former ${ }^{63}$. The type 2 CALR mutations are preferentially associated with ET, whereas type 1 predominates in PMF. The above three driver mutations are listed as major criteria for PV (JAK2V617F and exon 12), ET, and PMF (JAK2V617F, CALR and MPL) in the revised 2016 classification $^{1,51}$. Therefore, the modern diagnostic approach to MPN requires the knowledge of mutation status ${ }^{64}$. However, in the instances when genotyping for these mutations is not available, or the mutations result absent in diagnostic samples, minor criteria in the WHO classification are included to support diagnosis otherwise. Some PV patients who lack JAK2 mutations might eventually harbor other mutations in 
$J A K 2^{64}$ or other genes such as $S H 2 B 3 / L N K^{65}$. On the other hand, up to $20 \%$ of ET and $10-15 \%$ of PMF patients have no driver mutations, and are currently referred as "triple-negative" (TN); some of these case have noncanonical mutations in $M P L$ and JAK2, but overall they do not account for $>10 \%$ of the TN category ${ }^{66,67}$. For triplenegative PMF patients, the 2016 WHO classification supports the search for other non-driver "most frequent" mutations, e.g., in ASXL1, EZH2, TET2, IDH1/IDH2, $S R S F 2$, SF3B1, that if present stand as a marker of clonality. These mutations lack both disease specificity and mutual exclusivity; however, they are found in $~ 50 \%$ of cases with PMF $^{14,68}$ and using wider amplicon panels up to $81 \%$ of the patients presented one clonal marker ${ }^{69}$. Although not explicitly stated in the WHO classification, also chromosomal abnormalities might serve as marker of clonality. Presence of the above additional mutations is not currently included as criteria of clonality in cases of PV or ET lacking driver mutations, although a recent large study showed that $~ 50 \%$ of the patients had at least one such mutations ${ }^{70}$. Interpretation of these genetic variants is complicated by the discovery of CHIP, "clonal hematopoiesis of indeterminate potential", that reflects the "trending toward inevitability" ${ }^{\text {"11 }}$ age-related accumulation of mutations ${ }^{72,}{ }^{73}$; however, in the context of hematologic abnormalities that characterize MPN patients, finding any of these mutations certainly is in favor of the existence of a pathologic clonal hematopoiesis.

\section{Current controversies regarding the new diagnostic criteria, especially in regards to diagnosis of PV and prefibrotic PMF}

Serious concern has been expressed by several authors regarding the lowering of the diagnostic $\mathrm{Hb}$ threshold values $(>16.5 \mathrm{~g} / \mathrm{dL}$ for men and $>16.0 \mathrm{~g} / \mathrm{dL}$ for women) proposed by the 2016 revision by the $\mathrm{WHO}^{1,2}$ for the diagnosis of $\mathrm{PV}^{36,74,75}$. The main points of criticism are that these new criteria will lead to unnecessary and costly investigations including a large segment of the healthy population $^{36,74}$. To evaluate the proportion of presumptive PV by strict application of the 2016 WHO criteria regarding the low $\mathrm{Hb}$ thresholds ${ }^{1,2}$, a retrospective analysis of the complete blood cell count (CBC) was performed on very large cohorts of unselected subjects ${ }^{36}$, 74 . Following this scotom-like focus on one single parameter gained from routinely done $\mathrm{CBCs}$ in the Canadian population $\sim 4.1 \%$ of the males and $0.35 \%$ of the females revealed these $\mathrm{Hb}$ threshold values ${ }^{36}$ compared with the Brazilian population with $\sim 5.6 \%$ males and $0.22 \%$ females ${ }^{75}$. These data would imply that the annual incidence of potential PV patients may increase by up to 12 fold in males and threefold in females ${ }^{36,74}$. However, it has to be noted that these data were derived from routinely performed $\mathrm{CBCs}$, without any knowledge about the JAK2 mutation status and were not obtained from clinic-pathological databases as the WHO threshold values for $\mathrm{Hb}^{74}$.

In contrast, patients presenting with $\mathrm{mPV}^{30,} 33$ showed that many cases as defined by the WHO 2016 criteria $^{1,2}$ were actually missed. A study on 118 patients with mPV included $72 \%$ cases with a history of previous arterial and /or venous thrombosis and according to the applied CBC parameters showed thrombocytosis as being the most frequent finding with $64 \%$ (either isolated or combined with leukocytosis $)^{34}$.

Thrombocytosis presents an important issue concerning the differentiation between $\mathrm{mPV}$ and $\mathrm{ET}^{50,51}$ that has been already recognized before the establishment of the 2016 WHO revision ${ }^{1,2}$ and was further emphasized regarding therapeutic consequences ${ }^{76}$. Misdiagnosis of $\mathrm{mPV}$ for ET implies that phlebotomies will erroneously not be considered ${ }^{36}$. In this context it should be underscored that PV patients require phlebotomies to a therapeutic Hct target of $<45 \%{ }^{77,78}$. Summarized, recognition of early stages of PV is in keeping with a major advancement in the field of MPNs and will certainly avoid underdiagnosis by preventing fatal thrombotic events and initiation of proper treatment ${ }^{30,35,36,78}$.

Current problems associated with pre- PMF and ET start with the fact that existence of a pre-PMF is not everywhere recognized, although as the first descriptions in the late nineties ${ }^{13}$ its existence including its clear differentiation from $\mathrm{ET}^{79,80}$ has been demonstrated. Following a lively discussion in the past years ${ }^{38}$ pre-PMF was definitely confirmed by several groups ${ }^{14,15,38,81-85}$ but until now not regarded by the updated British guidelines ${ }^{16,86}$. According to the 2008/2016 WHO classification $^{1,2,12}$ pre-PMF may present either with no increase (fiber grade 0 ) or minor grade of reticulin fibrosis (fiber grade 1$)^{87}$, whereas overt (classical) PMF is characterized by fiber grades 2 and 3 including collagen ${ }^{88}$. Difficulties to accept pre-PMF as clinically relevant entity may be caused by the fact that diagnosis of pre-PMF was predominantly based on morphological characteristics and that presenting clinical features may be different depending whether pre-PMF patients were collected from cohorts with an ET-like phenotype $\mathrm{e}^{15,17,18}$ or with features resembling a more PMF-like phenotype ${ }^{85}$ without thrombocytosis ${ }^{14}$. Molecular markers of pre-PMF are different from ET, but their discriminant power is relatively low ${ }^{13,} 26$.

Recent investigations confirm that clinical presentation of pre-PMF is different from ET and this may influence therapeutic decision making and outcome. Ample evidence has been provided by several groups that an accurate discrimination between pre-PMF and ET is not 
trivial $^{13}$ but has an impact not only on presenting laboratory data but also on complications like disposition to hemorrhage, thrombosis, and outcome with progression to overt myelofibrosis, transformation to blast crisis, and overall survival ${ }^{15-18,32}$.

Laboratory data at initial diagnosis are of distinctive impact between pre-PMF and WHO-defined ET as it is shown that at least one of the minor criteria for diagnosis of pre-PMF defined by the WHO (anemia, leukocytosis, elevated LDH levels, and splenomegaly) is highly prevalent with $91 \%$ in pre-PMF in comparison with $48 \%$ in $\mathrm{ET}^{15}$. Greater values of circulating CD34 cell count in prePMF in comparison with ET and a significantly more active in vitro stem growth in peripheral blood MNCs from pre-PMF are valid parameters for a different biological behavior ${ }^{15,17,18,38,83,89}$. This is in line with the observation of a prognostic unfavorable impact of the $J A K 2 \mathrm{~V} 617 \mathrm{~F}$ mutation in pre-PMF versus a more benign course of disease in patients with a CALR mutation, which could not be seen in ET patients strictly diagnosed by WHO criteria ${ }^{15}$.

To investigate if blood tests can exert a predictive power in patients presenting clinically with an ET-like phenotype $\mathrm{Hb}$ value, WBC count and LDH level were used in a dichotomized fashion, resulting in a step-by-step procedure. Utilizing this algorithm provided a sensitivity and specificity of $\sim 50 \%{ }^{90}$. To confirm and improve this investigation by expanding the so-called Bergamo algorithm regarding its discriminatory ability, a novel logistic regression model was introduced generating a substantial increase in sensitivity and specificity to $\sim 75 \%{ }^{91}$. In aggregate the authors of this investigation concur that although BM biopsy examination persists to remain an integral part of the final diagnosis, laboratory parameters at presentation may provide clinicians with additional information to suspect pre-PMF in a patient with a presumptive clinical diagnosis of ET.

Regarding the rates for survival, blast transformation (acute leukemia), and progression to overt myelofibrosis data were significantly worse in pre-PMF compared to WHO-confirmed $\mathrm{ET}^{13-15}, 17,18,38,92-94$. Moreover, the striking differences in clinical phenotypes between prePMF and overt PMF may not allow to use the risk scoring systems established for overt PMF for decision making in pre-PMF ${ }^{84}$.

Finally, the different clinical picture and outcomes in pre-PMF and ET result in different treatment needs. This is impressively demonstrated by different treatment outcomes when hydroxyurea was prospectively compared with anagrelide in ET patients diagnosed according to the PVSG criteria (designating many pre-PMF patients as ET) with an advantage for hydroxyurea in the UK-PT1 study versus the same comparison in WHO-classified ET in the anahydret study with an equal efficacy of anagrelide ${ }^{21,95}$.

\section{Ongoing controversies with regard to BM morphology in the diagnosis of MPN subtypes}

It has been argued that performing a BM trephine biopsy in JAK2-mutated patients with sustained absolute erythrocytosis with $\mathrm{Hb}$ concentrations of $>18.5 \mathrm{~g} / \mathrm{dL}$ in men or $>16.5 \mathrm{~g} / \mathrm{dL}$ in women or Hct $>55.5 \%$ in men or $>49.5 \%$ in women, might be associated with some hazards for the patient and is not warranted. In addition, it has been argued that morphology in general does not provide enough diagnostic specificity for the differentiation of PV from other types of MPN, nor does provide useful prognostic information ${ }^{49}$. The concerns in relation to complications related to BM biopsy seems to be unsubstantiated ${ }^{96}$.

A recent blinded review study has shown that characteristic BM features of PV are highly reproducible with an overall interobserver agreement of almost $93 \%{ }^{39}$. Interestingly, this series did include specimens of $\mathrm{mPV}$, overt PV, and JAK2-mutated ET, as well as other JAK2mutated patients that did not meet the 2008 WHO threshold ${ }^{12}$ for an elevated Hb level but were confirmed as PV based on their increased red cell mass ${ }^{40}$. BM biopsy is also capable of providing prognostic information. This is particularly true for the identification of BM fibrosis ${ }^{87,88}$. Although a variable incidence and severity of BM fibrosis has been reported in the past, it has to be emphasized that most of these older studies included advanced disease stages more consistent with post-PV myelofibrosis ${ }^{97}$ presenting with grades 2 and 3 of reticulin/collagen fibrosis ${ }^{98,99}$. The clinical impact and prognostic relevance of the presence at disease outset of reticulin fibrosis ${ }^{38}$ has been demonstrated in $>500$ patients with WHO-defined PV who were strictly evaluated at time of initial diagnosis. In this study, grade 1 reticulin fibrosis ${ }^{87}$ was found in $14 \%$ of patients and in only two cases a higher grade could be observed $^{100}$. In general, clinical and laboratory characteristics did not differ between patients with or without $\mathrm{BM}$ fibrosis, however, a significant higher prevalence of palpable splenomegaly was observed in cases with BM fibrosis, and most importantly, patients presenting with initial fibrosis transformed more frequently into post-PV myelofibrosis $^{100}$. These data were recently validated by emphasizing the association between BM reticulin fibrosis at onset of PV and subsequent fibrotic progression ${ }^{101}$. In addition, palpable splenomegaly and leukocytosis were also identified as important risk factors ${ }^{101}$. For this reason, evaluation of a BM biopsy specimen in PV validates not only the accurate diagnosis, especially in doubtful cases ${ }^{78}$, but also provides important information concerning progression to post-PV myelofibrosis (spent phase). Altogether, the recognition that PV is characterized by a specific histological BM pattern ${ }^{37-40,92,102}$, allowed the "promotion" of BM histology to one of the major diagnostic criteria in the 2016 WHO revision ${ }^{1,2}$. Accordingly, 
BM biopsy examination was recommended to be performed in a recently published practical diagnostic algorithm for PV and secondary polycythemia ${ }^{78}$.

Discussion and controversies persists that histological criteria characterizing the specific MPN subtypes of prePMF and ET, as described by the WHO classification ${ }^{1,2,12 \text {, }}$ 103 are difficult to apply, and thus unreliably reproducible in routine practice. It has been postulated that a more objective, algorithmic-based procedural approach that also include a quantitative assessment of individual morphological features ${ }^{13,}{ }^{41}$ should instead be applied to achieve a clearer separation of true ET from pre-PMF. It should be noticed, however, that the diagnosis of specific subtypes, in particular in early stages, is not captured by single morphological parameters ${ }^{92}$, but must takes into account the entirety of the complex BM architecture in MPN, which is best captured by specific diagnostic patterns $^{21,}{ }^{43}$. In relation to PMF, it is important to realize that grading of $\mathrm{BM}$ fibrosis has a significant impact on clinical presentation and overall outcome $e^{14,104,} 105$. Moreover, regarding ET a major advancement of the 2016 WHO revision ${ }^{1,2}$ was to clarify incidence and the maximum grade of reticulin fibrosis seen in this disease at its outset to strengthen the differentiation from $\mathrm{PMF}^{15,18,38}$. Reproducibility of WHO-defined morphological features for the differentiation of ET from pre-PMF has been evaluated by studying large cohorts of patients with varying numbers of involved panelists with or without prior knowledge of clinical data. In aggregate, $>80 \%$ (range 76-88\%) diagnostic consensus with formal assessment of interobserver variability was reached in 2033 patients derived from several independent study groups ${ }^{17-}$ 19,45 . It has to be stressed that for the first time in one of these studies, specimens representing a wide spectrum of reactive lesions as well as normal BM and all major subtypes of MPNs were included to more closely reflect a "real world" pathology setting, i.e., daily routine ${ }^{19}$. Referring to the reliability to reproduce the postulated WHO guidelines ${ }^{1,2,} 12$ the group of unclassifiable MPNs (MPN$\mathrm{U})$ has to be briefly discussed herewith. The proportion of cases that a given pathology deem to be "unclear" and thus allocates to the MPN-U group, may be considered as a true yardsticks for the accuracy to discriminate MPN subtypes. Reported incidence of MPN-U varies significantly in different studies with a range up to $>20 \%{ }^{19}$, ${ }^{106}$. However, most studies show an incidence of $10-15 \%$ or even less ${ }^{19,38}, 107$. When the 2016 WHO criteria ${ }^{1,2}$ have been applied the incidence is reduced to $<5 \%{ }^{47}$. These conspicuous differences may be significantly related to the differences in experience of the reviewer, a high incidence of cases of MPN presenting in very early phase, preceding cytoreductive treatment which may have affected the morphologic findings and/or incomplete clinical data and mutation status knowledge ${ }^{92}$.

\section{Challenges in applying the WHO criteria in routine clinical practice, and possible future directions}

The 2016 revised WHO classification ${ }^{1,2}$ is supposed to have immediate routine application, in particular regarding the early diagnosis of PV and a clearer-cut distinction between pre-PMF and both ET and overt PMF, as such distinction has important outcome correlates ${ }^{14,15,85}$. The value to recognize early and distinct phases of diseases, through the characterization of as homogeneous as possible clinical, histopathology and molecular patterns (for example, CALR mutation is very unlikely to indicate PV, if not exceptionally ${ }^{108}$, and MPL mutation virtually negates it), is projected to improve the management and hopefully the outcome.

With this in mind, we think that the adoption of the revised 2016 WHO criteria $^{1,2}$ in the clinical practice as a "state-of-the-art" approach, yet in an ever changing research scenario, will be the best way to collect homogenously defined categories of patients for assessing their clinical course, outcome, response to conventional and new target therapies and, not by least, provide material for further molecular and cellular studies aimed at discovering surrogate diagnostic biomarkers. Gene and/or noncoding small RNA expression profiles in the context of selected mutation patterns, Nano-String interrogation of BM tissues, levels and types of inflammatory cytokines and chemokines, cell membrane antigen combinations, are all fields of investigation that have the chance to delineate integrated patterns. These novel techniques may eventually replace BM biopsies that, however, presently stand as a stone regarding the modern diagnostic approach to MPN.

\section{Challenges in applying the WHO criteria in routine clinical practice-future directions from the perspective of the hematopatholgist}

The reproducibility of the histological characteristics as described in the WHO classification remains a debate issue $^{49}$. Although the overall histological evaluation shows a high degree of reproducibility, the identification of specific morphological features displays a more limited reproducibility among different hemato-pathologists. The level of consensus has revealed a wide range between 49 and $100 \%$ in some studies ${ }^{45}$. Several reasons acting alone or in concert may be hypothesized to account for these shortcomings: (1) failure to reproducibly identify standard BM features of distinctive diagnostic value ${ }^{43,44}$ undermining a correct morphological interpretation; $;^{17,19,39,} 92$ (2) inclusion of small, non-representative biopsy specimens with extensive crushing artefacts or fragmentation; (3) disregard of age-related adjustment for assessing hematopoietic cellularity ${ }^{87}$; (4) inability of performing an accurate fiber grading owing to a variety of staining $\operatorname{artefacts}^{86,106}$; (5) unexperienced investigators ${ }^{45}$. 
A central pathology review may be desirable in some clinical settings. Although BM fibrosis grading is considered a part of a standard BM biopsy examination report, low overall level of concordance of only 55.8\% (range 33-100\%) on 579 biopsy specimens between the local pathologists from various countries and a central review evaluation was demonstrated by a recent study ${ }^{109}$. This is in sharp contrast with central pathology review rates of $83-99.7 \%$; these rates of $>80 \%$ are considered to represent excellent agreement according to the standards used to measure the strength of concordance ${ }^{46}$.

All those issues are resolvable. In this regard, it has been demonstrated that the weighting of individual features defining a morphological pattern can be substantially affected by training sessions ${ }^{45}$. Educational seminars and workshops for hemato-pathologists can significantly improve the integration of all histological characteristics into a meaningful, reproducible subtyping of $\mathrm{MPNs}^{20,45}$. This includes an increased consensus on the identification of pre-PMF ${ }^{84}$.

In addition to distinguishing between the different subtypes of MPNs, their separation from MDS/MPN overlap syndromes or MDS particularly in clonally undefined (triple-negative) PMF has proven to be of upmost clinical importance as the outcome for the different subtypes varies significantly ${ }^{14,47,}, 92$.

\section{Conclusions}

The WHO committee of hemato-pathologists, clinicians, and scientists with special interest in MPN has now delivered the most comprehensive and practically useful outline of diagnostic criteria for ET, PV, and PMF. The authors of the current review strongly recommend the collection of BM examination at time of diagnosis of MPN and encourage repeating the procedure during follow-up, in the presence of signs of progressive disease. In all instances, sufficient BM aspiration should be secured in order to allow screening for driver and other mutations, as well as cytogenetic analysis.

Special attention to morphology is required in order to distinguish ET from pre-PMF and JAK2-mutated ET from PV. Such details are prognostically relevant as survival has been shown to be the longest in strictly WHO-defined ET, whereas it was significantly worse in pre-PMF and PV. $\mathrm{BM}$ examination is also the most optimal method of obtaining cytogenetic information that has been shown to influence survival in both PMF and PV. Establishing driver mutational status in patients with MPN is not only important in complementing morphologic diagnosis but also provides important prognostic information.

In regards to diagnosis, $\mathrm{PV}$ is expected to be almost always accompanied by a JAK2 mutation, whereas the specific driver mutation cannot otherwise distinguish one MPN from another; however, in distinguishing ET from
pre-PMF or $\mathrm{mPV}$, a higher $J A K 2 \mathrm{~V} 617 \mathrm{~F}$ allele burden favors the diagnoses of the latter rather than the former. In terms of prognosis, thrombosis risk in ET is strongly tied to the presence of JAK2 mutations, whereas the presence of type 1/like CALR mutations in PMF portends superior survival. In the future, we expect an increasing role for other mutations in complementing morphologic diagnosis in MPN and providing additional prognostic information.

\section{Author details}

${ }^{1}$ FROM Research Foundation, Papa Giovanni XXIII Hospital, Bergamo, Italy. ${ }^{2}$ Institute of Pathology, University of Cologne, Cologne, Germany. ${ }^{3}$ Medical University of Vienna, Vienna, Austria. ${ }^{4}$ Senckenberg Institute of Pathology, University of Frankfurt, Frankfurt, Germany. ${ }^{5}$ University of Florence, Florence, Italy. ${ }^{6}$ CRIMM-Centro Ricerca e Innovazione delle Malattie Mieloproliferative, Azienda Ospedaliera-Universitaria Careggi, Department of Experimental and Clinical Medicine, University of Florence, Florence, Italy. ${ }^{7}$ Department of Pathology and Laboratory Medicine, Weill Cornell Medical College, New York, NY, USA. ${ }^{8}$ Mayo Clinic, Rochester, MN, USA

\section{Conflict of interest}

The authors declare that they have no conflict of interest.

Received: 9 October 2017 Revised: 24 November 2017 Accepted: 5 December 2017

Published online: 09 February 2018

\section{References}

1. Swerdlow, S. H. et al. (eds). WHO classification of Tumours of Haematopoietic and Lymphoid Tissues. 4th edn, (International Agency for Research on Cancer, Lyon, France, 2017)

2. Arber, D. A. et al. The 2016 revision to the World Health Organization classification of myeloid neoplasms and acute leukemia. Blood 127, 2391-2405 (2016).

3. Bornhäuser, M. et al. Concurrent JAK2(V617F) mutation and BCR-ABL translocation within committed myeloid progenitors in myelofibrosis. Leukemia 21, 1824-1826 (2007).

4. Hussein, $\mathrm{K}$. et al. Myelofibrosis evolving during imatinib treatment of a chronic myeloproliferative disease with coexisting BCR-ABL translocation and JAK2V617F mutation. Blood 109, 4106-4107 (2007).

5. Maxson, J. E. et al. Oncogenic CSF3R mutations in chronic neutrophilic leukemia and atypical CML. N. Engl. J. Med. 368, 1781-1790 (2013).

6. Pardanani, A. et al. CSF3R T618l is a highly prevalent and specific mutation in chronic neutrophilic leukemia. Leukemia 27, 1870-1873 (2013).

7. Granger, J. M. \& Kontoyiannis, D. P. Etiology and outcome of extreme leukocytosis in 758 nonhematologic cancer patients: a retrospective, singleinstitution study. Cancer 115, 3919-3923 (2009).

8. Bain, B. et al. Chronic eosinophilic leukemia and the hypereosinophilic syndrome. In: E. S. Jaffe, N. L. Harris, H. Stein, J. W. Vardiman (eds). World Health Organization Classification of Tumours: Tumours of the Haematopoietic and Lymphoid Tissues (pp. 29-31. International Agency for Research on Cancer (IARC) Press, Lyon, France, 2001).

9. Wang, S. A. et al. Targeted next-generation sequencing identifies a subset of idiopathic hypereosinophilic syndrome with features similar to chronic eosinophilic leukemia, not otherwise specified. Mod. Pathol. 29, 854-864 (2016).

10. Pardanani, A. et al. Predictors of survival in WHO-defined hypereosinophilic syndrome and idiopathic hypereosinophilia and the role of next-generation sequencing. Leukemia 30, 1924-1926 (2016).

11. De Stefano, V. et al. Splanchnic vein thrombosis in myeloproliferative neoplasms: risk factors for recurrences in a cohort of 181 patients. Blood Cancer J. 6, e493 (2016). 
12. Vardiman, J. W. et al. The 2008 revision of the WHO classification of myeloid neoplasms and acute leukemia: rationale and important changes. Blood $\mathbf{1 1 4}$ 937-951 (2009).

13. Barosi, G. Essential thrombocythemia vs. early/prefibrotic myelofibrosis: why does it matter. Best. Pract. Res. Clin. Haematol. 27, 129-140 (2014).

14. Guglielmelli, P. et al. Presentation and outcome of patients with 2016 WHO diagnosis of prefibrotic and overt primary myelofibrosis. Blood 129, 3227-3236 (2017).

15. Jeryczynski, G. et al. Pre-fibrotic/early primary myelofibrosis vs. WHO-defined essential thrombocythemia: the impact of minor clinical diagnostic criteria on the outcome of the disease. Am. J. Hematol. 92, 885-891 (2017).

16. Gisslinger, $\mathrm{H}$. et al. Clinical impact of bone marrow morphology for the diagnosis of essential thrombocythemia: comparison between the BCSH and the WHO criteria. Leukemia 30, 1126-1132 (2016).

17. Thiele, J. et al. Essential thrombocythemia versus early primary myelofibrosis: a multicenter study to validate the WHO classification. Blood 117, 5710-5718 (2011).

18. Barbui, T. et al. Survival and disease progression in essential thrombocythemia are significantly influenced by accurate morphologic diagnosis: an international study. J. Clin. Oncol. 29, 3179-3184 (2011).

19. Gianelli, U., Iurlo, A., Cattaneo, D. \& Lambertenghi-Deliliers, G. Cooperation between pathologists and clinicians allows a better diagnosis of Philadelphia chromosome-negative myeloproliferative neoplasms. Expert Rev. Hematol. 7 255-264 (2014)

20. Madelung, A. B. et al. World Health Organization-defined classification of myeloproliferative neoplasms: morphological reproducibility and clinical correlations-the Danish experience. Am. J. Hematol. 88, 1012-1016 (2013).

21. Gisslinger, $\mathrm{H}$. et al. Anagrelide compared with hydroxyurea in WHO-classified essential thrombocythemia: the ANAHYDRET Study, a randomized controlled trial. Blood 121, 1720-1728 (2013)

22. Finazzi, G. et al. Incidence and risk factors for bleeding in 1104 patients with essential thrombocythemia or prefibrotic myelofibrosis diagnosed according to the 2008 WHO criteria. Leukemia 26, 716-719 (2012).

23. Barbui, T., Thiele, J. \& Tefferi, A. Myeloproliferative neoplasms. N. Engl. J. Med. 377, 894-895 (2017).

24. Campbell, P. J. et al. Definition of subtypes of essential thrombocythaemia and relation to polycythaemia vera based on JAK2 V617F mutation status: a prospective study. Lancet 366, 1945-195 (2005).

25. Rumi, E. et al. JAK2 or CALR mutation status defines subtypes of essential thrombocythemia with substantially different clinical course and outcomes. Blood 123, 1544-1551 (2014).

26. Tefferi, A., Guglielmelli, P. \& Larson, D. R. et al. Long-term survival and blast transformatioin molecularly annotated essential thrombocythemia, polycythemia vera, and myelofibrosis. Blood 124, 2507-2513 (2014).

27. Barbui, T., Thiele, J., Carobbio, A., Vannucchi, A. M. \& Tefferi, A. The rate of transformation from JAK2-mutated ET to PV is influenced by an accurate WHO-defined clinico-morphological diagnosis. Leukemia 29, 992-993 (2015).

28. Tefferi, A. et al. Calreticulin mutations and long-term survival in essential thrombocythemia. Leukemia 28, 2300-2303 (2014).

29. Rotunno, G. et al. Impact of calreticulin mutations on clinical and hematological phenotype and outcome in essential thrombocythemia. Blood 123 1552-1555 (2014).

30. Barbui, T. et al. Masked polycythemia vera (mPV): results of an international study. Am. J. Hematol. 89, 52-54 (2014).

31. Vainchenker, W. \& Kralovics, R. Genetic basis and molecular pathophysiology of classical myeloproliferative neoplasms. Blood 129, 667-679 (2017).

32. Barbui, T. et al. The 2016 revision of WHO classification of myeloproliferative neoplasms: clinical and molecular advances. Blood. Rev. 30, 453-459 (2016).

33. Alvarez-Larran, A. et al. Masked polycythaemia vera: presenting features, response to treatment and clinical outcomes. Eur. J. Haematol. 96, 83-89 (2016)

34. Barbui, T. et al. Diagnostic impact of the 2016 revised WHO criteria for polycythemia vera. Am. J. Hematol. 92, 417-419 (2017).

35. Lussana, F. et al. A lower intensity of treatment may underlie the increased risk of thrombosis in young patients with masked polycythaemia vera. $\mathrm{Br}$. J. Haematol. 167, 541-546 (2014).

36. Éthier V., Sirhan S., Olney H. J., Gupta V. \& Busque L. The 2016 WHO criteria for the diagnosis of Polycythemia Vera: benefits and potential risks. [e-letters]. Blood http://www.bloodjournal.org/content/127/20/2391/tab-e-letters.

37. Thiele, J. \& Kvasnicka, H. M. Diagnostic impact of bone marrow histopathology in polycythemia vera (PV). Histol. Histopathol. 20, 317-328 (2005).
38. Barbui, T., Thiele, J., Vannucchi, A. M. \& Tefferi, A. Myeloproliferative neoplasms: morphology and clinical practice. Am. J. Hematol. 91, 430-433 (2016)

39. Kvasnicka, H. M. et al. European LeukemiaNet study on the reproducibility of bone marrow features in masked polycythemia vera and differentiation from essential thrombocythemia. Am. J. Hematol. 92, 1062-1067 (2017).

40. Silver, R. T., Chow, W., Orazi, A., Arles, S. P. \& Goldsmith, S. J. Evaluation of WHO criteria for diagnosis of polycythemia vera: a prospective analysis. Blood $\mathbf{1 2 2}$ 1881-1886 (2013).

41. Barosi, G. et al. Identifying and addressing unmet clinical needs in Ph-neg classical myeloproliferative neoplasms: a consensus-based SIE, SIES, GITMO position paper. Leuk. Res. 38, 155-160 (2014).

42. Brousseau, M. et al. Practical application and clinical impact of the WHO histopathological criteria on bone marrow biopsy for the diagnosis of essential thrombocythemia versus prefibrotic primary myelofibrosis. Histopathology 56, 758-767 (2010)

43. Thiele, J. \& Kvasnicka, H. M. Diagnostic differentiation of essential thrombocythaemia from thrombocythaemias associated with chronic idiopathic myelofibrosis by discriminate analysis of bone marrow features - a clinicopathological study on 272 patients. Histol. Histopathol. 18, 93-102 (2003).

44. Thiele, J., Kvasnicka, H. M. \& Diehl, V. Standardization of bone marrow features - does it work in hematopathology for histological discrimination of different disease patterns? Histol. Histopathol. 20, 633-644 (2005).

45. Madelung, A. B. et al. WHO classification 2008 of myeloproliferative neoplasms: a workshop learning effect-the Danish experience. APMIS 123, 787-792 (2015)

46. Landis, J. R. \& Koch, G. G. The measurement of observer agreement for categorical data. Biometrics 33, 159-174 (1977).

47. Iurlo, A., Gianelli, U., Cattaneo, D., Thiele, J. \& Orazi, A. Impact of the 2016 revised WHO criteria for myeloproliferative neoplasms, unclassifiable: Comparison with the 2008 version. Am. J. Hematol. 92, E48-E51 (2017).

48. Rumi, E. \& Cazzola, M. Diagnosis, risk stratification, and response evaluation in classical myeloproliferative neoplasms. Blood 129, 680-692 (2017).

49. Spivak, J. L. Myeloproliferative neoplasms. N. Engl. J. Med. 376, 2168-2181 (2017).

50. Thiele, J., Kvasnicka, H. M. \& Diehl, V. Initial (latent) polycythemia vera with thrombocytosis mimicking essential thrombocythemia. Acta Haematol. 113 213-219 (2005).

51. Gianelli, U. et al. The significance of bone marrow biopsy and JAK2V617F mutation in the differential diagnosis between the "early" prepolycythemic phase of polycythemia vera and essential thrombocythemia. Am. J. Clin. Pathol. 30, 336-342 (2008).

52. James, C. et al. A unique clonal JAK2 mutation leading to constitutive signalling causes polycythaemia vera. Nature 434, 1144-1148 (2005).

53. Baxter, E. J. et al. Acquired mutation of the tyrosine kinase JAK2 in human myeloproliferative disorders. Lancet 365, 1054-1061 (2005).

54. Levine, R. L. et al. Activating mutation in the tyrosine kinase JAK2 in polycythemia vera, essential thrombocythemia, and myeloid metaplasia with myelofibrosis. Cancer Cell 7, 387-397 (2005).

55. Kralovics, R. et al. A gain-of-function mutation of JAK2 in myeloproliferative disorders. N. Engl. J. Med. 352, 1779-1790 (2005).

56. Scott, L. M. et al. JAK2 exon 12 mutations in polycythemia vera and idiopathic erythrocytosis. N. Engl. J. Med. 356, 459-468 (2007).

57. Pikman, $Y$. et al. MPLW515L is a novel somatic activating mutation in myelofibrosis with myeloid metaplasia. PLoS Med. 3, e270 (2006)

58. Passamonti, F. et al. Molecular and clinical features of the myeloproliferative neoplasm associated with JAK2 exon 12 mutations. Blood 117, 2813-2816 (2011)

59. Ding, J. et al. Familial essential thrombocythemia associated with a dominant-positive activating mutation of the c-MPL gene, which encodes for the receptor for thrombopoietin. Blood 103, 4198-4200 (2004).

60. Pardanani, A. D. et al. MPL515 mutations in myeloproliferative and other myeloid disorders: a study of 1182 patients. Blood 108, 3472-3476 (2006)

61. Klampfl, T. et al. Somatic mutations of calreticulin in myeloproliferative neoplasms. N. Engl. J. Med. 369, 2379-2390 (2013).

62. Nangalia, J. et al. Somatic CALR mutations in myeloproliferative neoplasms with nonmutated JAK2. N. Engl. J. Med. 369, 2391-2405 (2013).

63. Tefferi, A. et al. The prognostic advantage of calreticulin mutations in myelofibrosis might be confined to type 1 or type 1-like CALR variants. Blood 124, 2465-2466 (2014) 
64. Guglielmelli, P. et al. Recommendations for molecular testing in classical Ph1neg myeloproliferative disorders-A consensus project of the Italian Society of Hematology. Leuk. Res. 58, 63-72 (2017).

65. McMullin, M. F. \& Cario, H. LNK mutations and myeloproliferative disorders. Am. J. Hematol. 91, 248-251 (2016).

66. Cabagnols, $\mathrm{X}$. et al. Presence of atypical thrombopoietin receptor (MPL) mutations in triple-negative essential thrombocythemia patients. Blood 127, 333-342 (2016).

67. Milosevic Feenstra, J. D. et al. Whole-exome sequencing identifies novel MPL and JAK2 mutations in triple-negative myeloproliferative neoplasms. Blood 127, 325-332 (2016)

68. Vannucchi, A. M. et al. Mutations and prognosis in primary myelofibrosis. Leukemia 27, 1861-1869 (2013).

69. Tefferi, A. et al. Targeted deep sequencing in primary myelofibrosis. Blood Adv. 1, 105-111 (2016).

70. Tefferi, A. et al. Targeted deep sequencing in polycythemia vera and essential thrombocythemia. Blood Adv. 1, 21-30 (2016).

71. Zink, F. et al. Clonal hematopoiesis, with and without candidate driver mutations, is common in the elderly. Blood 130, 742-752 (2017)

72. Jaiswal, S. et al Age-related clonal hematopoiesis associated with adverse outcomes. N. Engl. J. Med. 371, 2488-2498 (2014)

73. Xie, M. et al. Age-related mutations associated with clonal hematopoietic expansion and malignancies. Nat. Med. 20, 1472-1478 (2014).

74. Busque, $L$. et al. Laboratory investigation of myeloproliferative neoplasms (MPNs): recommendations of the Canadian MPN Group. Am. J. Clin. Pathol. 146, 408-422 (2016).

75. Sandes, A. F. \& Gonçalves, M. V., \& Chauffaille, M. L. Frequency of polycythemia in individuals with normal complete blood cell counts according to the new 2016 WHO classification of myeloid neoplasms. Int. J. Lab. Hematol. 39, 528-531 (2017).

76. Barbui, T. et al. Discriminating between essential thrombocythemia and masked polycythemia vera in JAK2 mutated patients. Am. J. Hematol. 89, 588-590 (2014).

77. Marchioli, R. et al. CYTO-PV Collaborative Group. Cardiovascular events and intensity of treatment in polycythemia vera. N. Engl. J. Med. 368, 22-33 (2013).

78. Tefferi, A. \& Barbui, T. Polycythemia vera and essential thrombocythemia: 2017 update on diagnosis, risk-stratification, and management. Am. J. Hematol. 92, 94-108 (2017)

79. Thiele, J. et al. Primary (essential) thrombocythemia versus initial (hyperplastic) stages of agnogenic myeloid metaplasia with thrombocytosis--a critical evaluation of clinical and histomorphological data. Acta Haematol. 81 192-202 (1989).

80. Thiele, J. et al. Initial (prefibrotic) stages of idiopathic (primary) myelofibrosis (IMF) - a clinicopathological study. Leukemia 13, 1741-1748 (1999).

81. Florena, A. M. et al. Value of bone marrow biopsy in the diagnosis of essential thrombocythemia. Haematologica 89, 911-919 (2004).

82. Gianelli, U. et al. Essential thrombocythemia or chronic idiopathic myelofibrosis? A single-center study based on hematopoietic bone marrow histology. Leuk. Lymphoma 47, 1774-1781 (2006).

83. Barosi, G. et al. Evidence that prefibrotic myelofibrosis is aligned along a clinical and biological continuum featuring primary myelofibrosis. PLOS ONE 7. e35631 (2012).

84. Gisslinger, H. Pre-PMF emerging as important subgroup of MPN. Blood 129 3142-3144 (2017).

85. Mudireddy M. et al. Prefibrotic versus overtly fibrotic primary myelofibrosis: clinical, cytogenetic, molecular and prognostic comparisons. Br J Haematol (2017). https://doi.org/10.1111/bjh.14838. [Epub ahead of print]

86. Reilly, J. T. et al. Use of JAK inhibitors in the management of myelofibrosis: a revision of the British Committee for Standards in Haematology Guidelines for Investigation and Management of Myelofibrosis 2012. Br. J. Haematol. 167, 418-420 (2014)

87. Thiele, J. et al. European consensus on grading bone marrow fibrosis and assessment of cellularity. Haematologica 90, 1128-1132 (2005).

88. Kvasnicka, H. M. et al. Problems and pitfalls in grading of bone marrow fibrosis, collagen deposition and osteosclerosis - a consensus-based study. Histopathology 68, 905-915 (2016).
89. Geissler, K. et al. Circulating hematopoietic progenitor cells in essential thrombocythemia versus prefibrotic/early primary myelofibrosis. Am. J. Hematol. 89, 1157-1158 (2014).

90. Carobbio, A. et al. Blood tests may predict early primary myelofibrosis in patients presenting with essential thrombocythemia. Am. J. Hematol. 87, 203-204 (2012)

91. Schalling, M. et al Essential thrombocythemia versus prefibrotic/early primary myelofibrosis: discrimination by laboratory and clinical data. Blood Cancer J. 7 643-647 (2017).

92. Kvasnicka, H. M. WHO classification of myeloproliferative neoplasms (MPN): A critical update. Curr. Hematol. Malign. Rep. 8, 333-341 (2013).

93. Kvasnicka, H. M. \& Thiele, J. The impact of clinicopathological studies on staging and survival in essential thrombocythemia, chronic idiopathic myelofibrosis and polycythemia rubra vera. Semin. Thromb. Hemost. 32, 362-371 (2006).

94. Ejerblad, E. et al. Diagnosis according to World Health Organization determines the long-term prognosis in patients with myeloproliferative neoplasms treated with anagrelide: results of a prospective long-term follow-up. Hematology 18, 8-13 (2013).

95. Harrison, C. N. et al. Hydroxyurea compared with anagrelide in high-risk essential thrombocythemia. N. Engl. J. Med. 353, 33-45 (2005).

96. Bain, B. J. Bone marrow biopsy morbidity: review of 2003. J. Clin. Pathol. 58 406-408 (2005)

97. Barosi, G. et al. Proposed criteria for the diagnosis of post-polycythemia vera and post-essential thrombocythemia myelofibrosis: a consensus statement from the International Working Group for Myelofibrosis Research and Treatment. Leukemia 22, 437-438 (2008).

98. Abdulkarim, K. et al. The impact of peripheral blood values and bone marrow findings on prognosis for patients with essential thrombocythemia and polycythemia vera. Eur. J. Haematol. 86, 148-155 (2011)

99. Tang, G. et al. Characteristics and clinical significance of cytogenetic abnormalities in polycythemia vera. Haematologica 102, 1511-1518 (2017).

100. Barbui, T. et al. Initial bone marrow reticulin fibrosis in polycythemia vera exerts an impact on clinical outcome. Blood 119, 2239-2241 (2012).

101. Barraco, D. et al. Prognostic impact of bone marrow fibrosis in polycythemia vera: validation of the IWG-MRT study and additional observations. Blood Cancer J. 7, e538 (2017)

102. Thiele, J. et al. Polycythemia rubra vera versus secondary polycythemias. A clinicopathological evaluation of distinctive features in 199 patients. Pathol. Res. Pract. 197, 77-84 (2001).

103. Barbui, T., Thiele, J., Vannucchi, A. M. \& Tefferi, A. Rationale for revision and proposed changes of the $\mathrm{WHO}$ diagnostic criteria for polycythemia vera, essential thrombocythemia and primary myelofibrosis. Blood Cancer J. 5, e337 (2015).

104. Thiele, J. \& Kvasnicka, H. M. Grade of bone marrow fibrosis is associated with relevant hematological findings - a clinicopathological study on 865 patients with chronic idiopathic myelofibrosis. Ann. Hematol. 85, 226-232 (2006)

105. Vener, C. et al. Prognostic implications of the European consensus for grading of bone marrow fibrosis in chronic idiopathic myelofibrosis. Blood 111, 1862-1865 (2008).

106. Alvarez-Larran, A. et al. WHO-histological criteria for myeloproliferative neoplasms: reproducibility, diagnostic accuracy and correlation with gene mutations and clinical outcomes. Br. J. Haematol. 166, 911-919 (2014)

107. Gianelli, U. et al. The myeloproliferative neoplasms, unclassifiable: clinical and pathological considerations. Mod. Pathol. 30, 169-179 (2017)

108. Broséus, J., Park, J-H. Carillo, S, Hermouet, S. \& Girodon, F. Presence of calreticulin mutations in JAK2-negative polycythemia vera. Blood 124, 3964-3966 (2014)

109. Pozdnyakova, O. et al. The importance of central pathology review in international trials: a comparison of local versus central bone marrow reticulin grading. Leukemia 29, 241-244 (2015).

110. Tefferi, A. et al. Survival and prognosis among 1,545 patients with contemporary polycythemia vera: an international study. Leukemia 27, 1874-188 (2013) 